\title{
Efectividad de un programa de rehabilitación respiratoria en pacientes con esclerosis múltiple
}

\author{
Effectiveness of a respiratory rehabilitation program on \\ patients with multiple sclerosis
}

\author{
Yanely Real González, ${ }^{\mathrm{I}}$ Mayda Nelia López Hernández, ${ }^{\mathrm{II}}$ Roberto Díaz \\ Márquez, ${ }^{\text {III }}$ José Antonio Cabrera Gómez ${ }^{\text {IV }}$ \\ ILicenciada en Defectología. Centro Internacional de Restauración Neurológica \\ (CIREN). La Habana, Cuba. \\ IILicenciada en Logopedia. Doctora en Ciencias de la Educación. Centro \\ Internacional de Restauración Neurológica (CIREN). La Habana, Cuba. \\ IIILicenciado en Cultura Física Terapéutica. Doctor en Ciencias en Cultura Física. \\ Centro Internacional de Restauración Neurológica (CIREN). La Habana, Cuba. \\ ${ }^{\mathrm{IV}}$ Doctor en Medicina. Doctor en Ciencias Médicas Centro Internacional de \\ Restauración Neurológica (CIREN). La Habana, Cuba.
}

\section{RESUMEN}

Introducción Las personas con esclerosis múltiple presentan alteraciones de la comunicación oral relacionadas con una insuficiencia respiratoria, lo que obliga a los especialistas en logopedia a trabajar en este problema desde la óptica de su especialidad.

Objetivo Evaluar la efectividad de un programa intensivo de rehabilitación respiratoria en personas con esclerosis múltiple, para aumentar su capacidad respiratoria y mejorar la coordinación fonorrespiratoria.

Métodos Estudio de intervención cuasi experimental en un grupo de 30 personas adultas de ambos sexos con esclerosis múltiple. Todos recibieron el programa durante cuatro semanas con una hora diaria de ejercitación. Para valorar la efectividad del programa, se realizó una evaluación inicial y otra final de la capacidad vital inspiratoria y espiratoria forzada y de la coordinación fonorrespiratoria, por personal externo. El deterioro neurológico se midió mediante la Escala Expandida de Discapacidad de Kurtzke.

Resultados En la muestra hubo predominio de mujeres, una edad promedio de $40,43 \pm 11,46$ años y 13,40 \pm 7,76 años de evolución de la enfermedad. Se encontró esclerosis múltiple progresiva en 22 pacientes y 8 tenían formas de brote-

http://scielo.sld.cu 
remisión. El deterioro neurológico fue de 5,8 $( \pm 1,51)$ como promedio, lo que habla a favor de una discapacidad moderada en la muestra. Se encontró un aumento de la capacidad vital inspiratoria y espiratoria forzada y un aumento en el tiempo máximo de fonación, en la emisión de series de palabras bisílabas y en la emisión de series de números.

Conclusiones El programa intensivo de rehabilitación respiratoria, contribuye al aumento de la capacidad respiratoria de las personas con esclerosis múltiple, por lo que se recomienda su aplicación en el proceso de neurorrehabilitación.

Palabras clave: Esclerosis múltiple, logopeda, rehabilitación respiratoria, comunicación.

\section{ABSTRACT}

Introduction People with multiple sclerosis present with altered oral communication related to respiratory failure, which forces the speech therapists to work on this problem within their range of specialty.

Objective To evaluate the effectiveness la efectividad) of an intensive respiratory rehabilitation program.

Methods Quasi-experimental interventional study carried out in a group of 30 adults of both sexes suffering from multiple sclerosis. All these patients were included in the program for 4 weeks, having one-hour training every day. For assessing the effectiveness of the program, an initial and a final evaluation of the forced vital inspiratory and expiratory capacity and of the phonorespiratory coordination was made by outside experts. The neurological deterioration was measured according the Kurtzke's extended disability scale.

Results Women predominated in the sample; the average age was $40.43 \pm 11.46$ years and progression of disease was $13.40 \pm 7.76$ years. Progressive multiple sclerosis was found in 22 patients and the onset-remission forms in 8 patients. The neurological deterioration amounted to $5.8( \pm 1.51)$ as an average, which speaks for the moderate disability rate present in the simple. There was observed increased vital forced inspiratory and expiratory capacities and increase in maximum phonation length and in pronouncing series of two-syllable words and series of numbers.

Conclusions The intensive respiratory rehabilitation program helps to increase the respiratory capacity of the patients with multiple sclerosis, hence, its implementation in the neurorehabilitation process is recommended.

Key words: Multiple sclerosis, speech therapist, respiratory rehabilitation, communication.

\section{INTRODUCCIÓN}

La historia natural de la esclerosis múltiple (EM) se caracteriza por un inicio con recaídas, en el 58-66 \% de los pacientes, que es la denominada forma de exacerbación-remisión (EM-ER). Estas recaídas van provocando deterioro y a los 10 años, la mitad de estas personas evolucionan a la forma secundaria progresiva

http://scielo.sld.cu 
(EM-SP). ${ }^{1,2}$ Una vez alcanzada la fase progresiva no hay ni recuperación ni remisiones espontáneas aunque algunos pacientes pueden tener largos periodos de estabilidad.

La EM puede también iniciarse con un curso progresivo, que se puede ver en el 18$34 \%$ de los pacientes, denominada forma primaria-progresiva (EM-PP). ${ }^{1,2} \mathrm{El}$ resultado final, en cuanto a deterioro y discapacidad, es que a los 20 años de padecer la enfermedad, las personas necesitan de algún apoyo y a los 30 años apenas pueden dar pasos. Otros sistemas funcionales como el visual, tallo cerebral, vesical, intestinal, sexual, sensoriales y cerebelar se van afectando progresivamente, lo que provoca grandes limitaciones a los enfermos.

Estos datos justifican la intervención con neurorrehabilitación en la EM, como un proceso que ayuda a la persona a alcanzar y mantener las máximas capacidades físicas, psicológicas, sociales, vocacionales y una calidad de vida consistente. Es por esto que la neurorrehabilitación debe ser considerada en todas las etapas de la enfermedad. ${ }^{1,3}$

La insuficiencia respiratoria, habitualmente se describe como un estado final de la EM con una letalidad de $8 \%$. La neumonía y los procesos respiratorios altos son las principales causas de morbilidad y mortalidad en estas personas. ${ }^{4}$ Sin embargo, en la mayoría de los casos, se brinda una escasa e inadecuada atención al aspecto progresivo del carácter restrictivo del componente respiratorio en la EM. La terapéutica generalmente comienza tardíamente cuando la falla respiratoria de tipo restrictivo se agrava con la aparición de algún componente obstructivo. ${ }^{5}$

Por otra parte, las alteraciones en el habla y la voz pueden acompañar el cuadro, pues estas funciones dependen principalmente de una adecuada actividad respiratoria, por lo que se hace necesario un temprano asesoramiento profesional y tratamiento continuo, lo que previene en el paciente con EM el desarrollo de insuficiencia respiratoria y secundariamente, problemas en la comunicación oral. Este estudio tiene como objetivo evaluar la efectividad de un programa intensivo de rehabilitación respiratoria en personas con esclerosis múltiple.

\section{MÉTODOS}

Es un estudio de intervención cuasi experimental en un grupo de 30 personas con EM según los criterios de McDonald y otros ${ }^{6}$ atendidos en la Clínica Raquimedular, Esclerosis Múltiple y Enfermedades Neuromusculares del Centro Internacional de Restauración Neurológica en el periodo 2006-2007. Se realizó una comparación del grupo antes y después de las intervenciones.

Los criterios de inclusión fueron: 1. Personas con EM según los criterios de McDonald y otros. ${ }^{6}$ 2. Todos los pacientes que estuvieran de acuerdo en participar en el estudio.

Los criterios de exclusión fueron: 1. Presencia de signos indicativos de deterioro mental o de complicaciones psiquiátricas en el momento del estudio. 2. Presencia de alguna contraindicación con la realización de un programa terapéutico intensivo. 3. Negativa del paciente a participar en el estudio.

Para medir los parámetros de efectividad se utilizaron como criterios primarios de

http://scielo.sld.cu 
respuesta: 1. La capacidad vital inspiratoria forzada (FIVC), que se evaluó en posición de sentado, mediante el inspirómetro manual con tres medidas: $600 \mathrm{cc}$, $900 \mathrm{cc}, 1200 \mathrm{cc}$ (TRIFLO III). 2. La capacidad vital espiratoria forzada (FVC), que se evaluó en posición de sentado, con el ESPIROTEST Modelo Kat. No. 2600. 3. La coordinación fonorrespiratoria, que fue evaluada en posición de sentado y se midió el tiempo máximo de fonación (TMF) y la cantidad de números y de palabras bisílabas que se pueden emitir con el uso de una espiración.

Las evaluaciones de los casos antes y después del tratamiento fueron realizadas por especialistas del Laboratorio de Evaluación Integral Psicomotor (LEIS), que no tienen relación con la terapia, quienes garantizaron la imparcialidad y el enmascaramiento en la evaluación.

El programa de rehabilitación respiratoria aplicado tuvo como objetivo: aumentar la capacidad respiratoria y mejorar la coordinación fonorrespiratoria. Las actividades realizadas fueron: fortalecimiento de músculos respiratorios, técnicas de control respiratorio, entrenamiento específico de los músculos espiratorios y ejercicios de coordinación fonorrespiratoria. Para este tratamiento se emplearon seis $\mathrm{h}$ semanales con una intervención de $24 \mathrm{~h}$ en un período de cuatro semanas por cada persona.

Para analizar los resultados de las variables cuantitativas se utilizó el cálculo de la media y la desviación estándar.

En la comparación de dos variables cuantitativas, se utilizó la T-Student y el test de Wilcoxon y para relacionar las variables se utilizó el coeficiente de correlación de Spearman con un nivel de significación de $5 \%$.

El deterioro neurológico se midió mediante la Escala Expandida de Discapacidad (EDSS) de Kurtzke.

\section{RESULTADOS}

De las 30 personas con EM incluidas en el estudio $22(73,3 \%)$ de la muestra tenían formas progresivas y $8(26,6 \%)$ tenían formas brote-remisión. El deterioro neurológico de la muestra medido por el EDSS fue de 5,8 $( \pm 1,51)$, se encontró predominio de mujeres $19(63,3 \%)$ y 11 hombres $(36,6 \%)$, edades medias 40,43 $\pm 11,46$ años y tiempo de evolución de la enfermedad de 13,40 $\pm 7,76$ años (tabla 1). 
Tabla 1. Variables demográficas y clínicas en personas con esclerosis múltiple

\begin{tabular}{|l|c|}
\hline Variables demográficas y clínicas & \multicolumn{1}{|l|}{} \\
\hline Género & Mujeres $19(63,3 \%)$ \\
\hline Edades (Media \pm DS) & Hombres $11(36,7 \%)$ \\
\hline Tiempo de evolución de la enfermedad en años (Media \pm DS) & $13,40 \pm 7,766(1-33)$ \\
\hline Personas con RR-MS & $8(26,6 \%)$ \\
\hline Personas con formas progresiva MS & $22(73,3 \%)$ \\
\hline Personas con primaria progresiva & $4(13,3 \%)$ \\
\hline Personas con secundaria progresiva & $18(60 \%)$ \\
\hline Deterioro (EDSS) & $5,8 \pm 1,5$ \\
\hline
\end{tabular}

Con relación con las variables de la coordinación fonorrespiratoria se observó un aumento del tiempo máximo de fonación (TMF), esta mejoría no fue estadísticamente significativa comparativamente antes y luego de 4 semanas de terapia: $14,1( \pm 6,6)$ al inicio versus $17( \pm 7,9) Z(-2,170) p=, 044$; se comportó de la misma manera en la emisión de series de palabras bisílabas: $16,6( \pm 11,5)$ al inicio versus $20,3( \pm 12) Z(-1,029) p=0,3$. En la emisión de series de números, se modificaron significativamente los resultados comparativamente antes y luego de la terapia: $37,9( \pm 31,6)$ inicio versus $42,9( \pm 29,8) Z(-3,223) p=0,001$ (tabla 2).

Tabla 2. Coordinación fonorrespiratoria. Evaluaciones antes y después del tratamiento

\begin{tabular}{|l|c|c|c|}
\hline \multirow{2}{*}{ Coordinación fonorrespiratoria } & $\begin{array}{c}\text { Inicial } \\
(n=30)\end{array}$ & $\begin{array}{c}\text { Final } \\
(n=30)\end{array}$ & Pruebas estadísticas \\
\cline { 2 - 2 } & Media $\pm \mathrm{DS}$ & Media $\pm \mathrm{DS}$ & \\
\hline Tiempo máximo de fonación & $14,1 \pm 6,1$ & $17,0 \pm 7,8$ & $\begin{array}{c}p=(0,04) \\
\text { Test de } \text { Wilcoxon }\end{array}$ \\
\hline Conteo numérico & $37,9 \pm 31,6$ & $42,9 \pm 29,8$ & $p=(0,001)$ \\
\hline Lectura de palabras bisílabas & $16,67 \pm 11,5$ & $20,3 \pm 12$ & $p=(0,3)$ \\
\hline
\end{tabular}

Las medidas de capacidad respiratoria se modificaron de manera significativa luego de 4 semanas de tratamiento. La capacidad vital inspiratoria forzada (CVIF) inicial fue de $1056,5 \mathrm{~mL}( \pm 177,9)$ versus $1202,6 \mathrm{~mL}( \pm 123,4) \mathrm{Z}(-2,714) p=0,007$; las evaluaciones de la capacidad vital espiratoria forzada (CVEF) inicial fue $2191,3 \mathrm{~mL}$ ( \pm 950$)$ versus $2500 \mathrm{~mL}( \pm 994) \mathrm{Z}(-2,614) p=0,016$.

\section{DISCUSIÓN}

Como habíamos planteado las personas con EM presentan una grave discapacidad neurológica fundamentalmente en las formas progresivas de la enfermedad, en este estudio, la gran parte de la muestra tiene formas progresivas lo cual indica que las personas con esta condición clínica acuden con mayor frecuencia a los servicios de neurorrehabilitación que las personas con brote-remisión. Somos del criterio que los programas de neurorrehabilitación son útiles en todas las etapas y formas clínicas de la enfermedad para mantener y alcanzar las máximas capacidades funcionales de estas personas, como sugiere el Dr. Cabrera JA, en el

http://scielo.sld.cu 
libro: Sclerosis for Practicing Neurologitst. ${ }^{7}$ Las personas tratadas tenían una discapacidad moderada medida por la escala de EDSS. Hubo predominio de mujeres, datos que coinciden con los informados en el estudio clínico y epidemiológico cubano realizado por Real González y otros en el año $2005 .^{8}$

En relación con las variables de la coordinación fonorrespiratoria, se observa un aumento del tiempo máximo de fonación (TMF), esta mejoría no es estadísticamente significativa comparativamente antes y luego decuatro semanas de terapia; se comporta de la misma manera en la emisión de series de palabras bisílabas. En la emisión de series de números se modifican significativamente los resultados comparativamente antes y luego de la terapia.

Para el habla es necesario un mayor control respiratorio que en la respiración fisiológica, se necesita de una mayor presión espiratoria y de un tiempo de fonación adecuado para lograr una buena proyección vocal. En la muestra estudiada no se encuentran cambios significativos, lo que pudiera estar dado porque en las evaluaciones iniciales estos parámetros están muy cerca de los rangos de normalidad, entonces la modificación significativa en ellos es mucho más difícil pues tendrían que variar más allá de la norma. Podemos decir entonces que en la muestra estudiada la baja capacidad respiratoria no interfirió en el control fonorrespiratorio.

En los pacientes tratados hubo un aumento tanto en la CVIF como en CVEF, lo que indica que la implementación de un programa de rehabilitación respiratoria favorece el aumento de los volúmenes pulmonares de estas personas.

Los resultados de este estudio coinciden con otros varios que utilizan el fortalecimiento muscular. ${ }^{9-11}$ El estudio más parecido metodológicamente a este es el de Klefbeck y otros en 15 personas con EM progresiva donde utilizaron un programa de fortalecimiento muscular por diez semanas y se obtuvo mejoría significativa en las evaluaciones de las presiones inspiratorias $(p<0,008)$ y espiratorias $(p<0,02),{ }^{9}$ lo que ayuda a confirmar que el procedimiento utilizado es efectivo. Además contamos con la ventaja de que el programa utilizado en ese estudio consume menos tiempo, lo quel disminuye los costos de la terapia.

Se puede concluir que el programa de rehabilitación intensiva durante cuatro semanas contribuye al aumento de la capacidad respiratoria en las personas con EM. Se recomienda como complemento en el proceso de neurorrehabilitación.

\section{REFERENCIAS BIBLIOGRÁFICAS}

1. Barnes MP, Gilhus NE, Wender M. Task force on minimum standards for health care of people with multiple sclerosis. Eur J Neurol. 2001;8:215-20.

2. Thompson A. Neurorehabilitation in multiple sclerosis: foundations, facts and fiction. Curr Opin Neurol. 2005;18:267-71.

3. Medical Advisory Board of the National Multiple Sclerosis Society. Rehabilitation: recomendetions for person with multiple sclerosis. [sitio en Internet] [citado 17 Jun 2005]. Disponible en:

http://www.nationalmssociety.org//pdf/forpros/ExpOp Rehab.pdf

http://scielo.sld.cu 
4. Binetty K. Pulmonary complications in the MS patient. MS Exchange. 2006;10(3):5-8.

5. Brown SA. Swallowing and speaking. Challengers for the Multiple Sclerosis patient. Int J MS Care. 2000;2:7-13.

6. McDonald WI, Compston A, Edan G. Recommended diagnostic criteria for multiple sclerosis: Guidelines from the International Panel on the Diagnosis of MS. Ann Neurol. 2001;50:121-7.

7. Cabrera Gómez JA. Neurorehabilitation in Multiple Sclerosis. En: Oger J, Al-Araji A, editors. Multiple Sclerosis for Practicing Neurologitst. New York: Demos Publishing Company. p. 75-85.

8. Real González Y, Cabrera Gómez JA, Romero García K. Epidemiological, clinical and socioeconomic study of Cuban women with MS. Inter J MS Care. 2005;15:1621.

9. Chira T, Martin D. Expiratory Muscle Strength Training in Persons With Multiple Sclerosis Having Mild to Moderate Disability: Effect on Maximal Expiratory Pressure, Pulmonary Function, and Maximal Voluntary Cough. Arch Phys Med Rehab. 2006;87:468-73.

10. Klefbeck B, Hamrah NJ. Effect of inspiratory muscle training in patients with multiple sclerosis. Arch Phys Med Rehab. 2003;84:994-9.

11. Fry DK, Pfalzer L A, Chokshi AR, Wagner MT, Jackson ES. Randomized Control Trial of Effects of a 10-Week Inspiratory Muscle Training Program on Measures of Pulmonary Function in Persons with Multiple Sclerosis. J Neurologic Physical Ther. 2007;31:162-2.

Recibido: 9 de febrero de 2010.

Aprobado: 30 de julio de 2010.

Yanely Real González. Centro Internacional de Restauración Neurológica (CIREN). Avenida 25 No. 5805 e/ 158 y 160, Reparto Cubanacán, Playa. La Habana, Cuba. Telf.: 271- 5044. Correo electrónico: yanelyreal@infomed.sld.cu 